\title{
ACUTE COMPLICATED PANCREATITIS AND DIABETES MELLITUS: THE ROLE AND SIGNIFICANCE OF THE BIOCHEMICAL INDICATORS OF CARBOHYDRATE AND LIPID METABOLISM AS A PROGNOSTIC CRITERION FOR THE SEVERITY OF THE DISEASE CLINICAL COURSE
}

10.36740/WLek202101104

\author{
Adrian D. Kvit, Mykhaylo M. Tutka, Volodymyr V. Kunovskiy \\ LVIV CLINICAL EMERGENCY HOSPITAL, LVIV, UKRAINE
}

\begin{abstract}
The aim: A clinical evaluation of biochemical parameters especially carbohydrate and lipid metabolism, their effect on the treatment program in patients with acute complicated pancreatitis.

Material and methods: The results of complex treatment of a sample group of 127 patients with acute pancreatitis were studied. A moderately severe clinical course of the disease was found in 42 patients (33.1\%) and severe in 85 patients (66.9\%), the ethanol genesis of the disease was stated in 73 (57.5\%), biliary genesis - in 54 (42.5\%) patients. The evaluation of the treatment effectiveness was based on the analysis of the dynamics of disease clinical manifestations and the monitoring of laboratory biochemical parameters and glucose levels

Results: It was stated, that the clinical course of the disease, severity, and prognosis of the patient's condition correlate with the dynamics of markers of endotoxicosis and hepatic insufficiency, and glucose level is one of the most sensitive criteria. The carbohydrate metabolism dynamics analysis in the blood of patients has shown that in most patients glucose levels increase notably with the increase in pancreatic swelling and reaches a peak by its destruction

Conclusions: The incidence of diabetes mellitus due to primary inflammation of the pancreatic parenchyma in ACP has a linear dependence on the frequency and duration of the disease; with the probability of developing diabetes with an exacerbation of the process during 5-8 years is $17.5 \%$. The level of blood glucose in patients with ACP can be a reliable indicator of the severity of the disease course and correlates with other indicators of metabolism
\end{abstract}

KEY WORDS: diabetes mellitus, Carbohydrate Metabolism, acute complicated pancreatitis, insulin dependent diabetes

Wiad Lek. 2021;74(1):22-27

\section{INTRODUCTION}

The epidemiology of secondary diabetes mellitus (DM) in the pathology of the pancreas, in particular in cases with pancreatitis, is not fully investigated. First of all, the reason for the complexity of diagnosis is that clear epidemiological data are not always amenable to statistical and clinical analysis. It is known that short-term hyperglycemia in acute pancreatitis (AP) develops in about half of patients who are hospitalized, and persistent hyperglycemia AP persists with a frequency of up to $15 \%[1,2,3,4,5]$.

It should be noted that short-term (transient) hyperglycemia also develops in case of chronic pancreatitis (CP) exacerbation, which in most cases is associated with pancreatic edema and the inhibitory effect of trypsin on insulin production, and its increase in the blood $[6,7,8,9,10]$.

In patients with AP, there is a tendency that is determined by metabolic disorders from carbohydrate metabolism. It was noted that usually in the first day after the disease appearance, blood glucose keeps at the level of reference values, and increases starting from 3-4 days. This is due to numerous stress-gene factors, which are usually affected by the severity of the underlying disease [11].

According to various sources, DM occurs in $15-18 \%$ of patients with AP and 10-90\% of patients with CP, and in half of cases, insulin-dependent diabetes (IDDM) develops [12]. Such a big difference in the literature data on the incidence of DM with AP and CP is associated both with poor diagnosis of the underlying disease and with a different degree of probability of developing endocrine disorders in various forms of pancreatitis. It should be noted that carbohydrate tolerance is usually observed already in the early stages of observation $[7,11,13,14]$. DM can also form at the beginning of the clinical manifestation of pancreatitis, but still more often, the persistent disorder of carbohydrate metabolism occurs in about 5 years after the onset of the underlying disease, with chronic inflammation and on the background of frequent remissions of AP (2-3 times a year), when development of DM becomes a linear time dependence [5].

According to some authors [12], in case of the manifestation of the disease (AP and exacerbation of $\mathrm{CP}$ ), $\mathrm{DM}$ is 


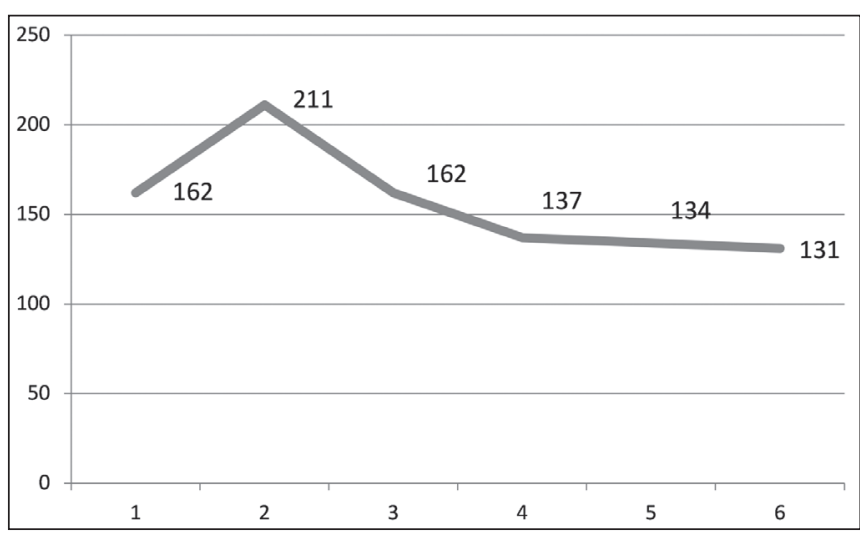

Fig.1. a-amylase level in the blood serum during treatment.

diagnosed in $10 \%$ of cases, and in $2 \%$ - IDDM. After 10 years from the onset of the underlying disease - in $50 \pm 3$ and $26 \pm 3 \%$ of cases. After 25 years, these indicators reach $83 \pm 4$ and $53 \pm 6 \%$. The annual probability of developing DM with pancreatitis of biliary origin is 3.5\%, and IDDM - $2.2 \%$. In almost $80-90 \%$ of patients whose pancreatic lesions were verified and at least one case in the year of its exacerbation, during 25 years the possibility of DM appearance is $65-85 \%$.

So, despite the fact that the features of the acute complicated pancreatitis (ACP) pathogenesis and clinical course have been widely studied by clinicians in European countries over the last few years, a number of questions remain unresolved $[5,9]$. This applies, in particular, to the unification of approaches in the clinical assessment of biochemical parameters in patients with ACP, taking into consideration their importance for predicting the course of the disease, the characteristics of the diagnostic algorithm and approaches to conservative treatment $[2,3]$.

\section{THE AIM}

The aim of the research, which is coming out of the above mentioned, is to study changes in carbohydrate and lipid metabolism in patients with ACP.

\section{MATERIALS AND METHODS}

The results of complex treatment of a sample group of patients with AP in the amount of 127 patients, who were hospitalized in the emergency hospital with a diagnosis of ACP were studied. In accordance with the criteria of Atlanta (2012) [3], a moderately severe clinical course of the disease was found in 42 patients (33.1\%) and severe in 85 patients (66.9\%). According to the pathogenesis of the disease in 73 (57.5\%) patients, the ethanol genesis of the disease was stated, in 54 (42.5\%) - biliary genesis.

In all patients, in the complex of conservative treatment, a unified diagnostic treatment algorithm was used, based on local treatment protocols for patients with AP and clinically tested approaches to the management of this patient group. All patients were randomized by age, sex, disease severity, degree of pancreatic lesion, parapancreatic / paracolary retroperitoneal fatty tissue and were treated in the intensive care unit for the first 7-14 days. Surgical treatment (including minimally invasive, puncture techniques) in the case of infected pancreatic necrosis and / or purulent-necrotic parapancreatitis / paracolitis was performed in $74(58.3 \%)$ patients.

Evaluation of the treatment effectiveness was done on the basis of an analysis of the disease clinical manifestations dynamics and laboratory biochemical parameters (carbohydrate, protein, lipid metabolism) monitoring. The obtained results were analysed by using MedStat programe for medical statistics information.

\section{RESULTS}

An analysis of the dynamics of laboratory indicators of blood sugar levels (carbohydrate metabolism) in patients with ACP showed that in most patients the blood glucose level increases markedly with an increase in pancreatic edema and reaches its maximum during its destruction.

In the time of hospitalization in almost all patients during first days of the disease, an increase in serum $\alpha$-amylase levels $(\mathrm{N}=30-130 \mathrm{U} / \mathrm{L})$ was detected, which confirmed inflammation of the pancreatic parenchyma - acute pancreatitis (Fig.1).

In 63 patients from the group of examined patients, during the first 12 hours of the disease, the serum glucose content varied within the upper normal range of $5.8 \pm 3.1$ $\mathrm{mmol} / \mathrm{L}$. Subsequently, in all patients, regardless of the pathological process nature and the degree of pancreatic parenchyma damage, an increase in glucose up to the level of $6.3 \pm 9.1 \mathrm{mmol} / \mathrm{l}$ was observed. In other patients, glucose growth was already observed in the first hours of the disease to levels of $10.4 \pm 3.2 \mathrm{mmol} / \mathrm{L}$.

After monitoring changes in serum glucose levels, certain regularity can be noted that allows us to establish a relationship between the degree of glucose growth and the severity of the disease, starting from the second day of treatment of patients with AP. This indicator, according to our observations, is a statistically significant marker for assessing the patient's condition and the effectiveness of the infusion therapy.

The analysis of changes in serum glucose levels allowed us to state a regularity, namely, at the beginning of the disease, glucose levels remain normal or moderate hyperglycemia $(5.7 \pm 1.3 \mathrm{mmol} / \mathrm{l})$ is stated, and statistically from the second or third day significant $(\mathrm{p}<0.05)$ increase in blood plasma glucose is observed. This is due to the activation of the insular apparatus functioning in the first hours of the disease and the inhibition of the gland islets activity with the development of destructive changes. In cases with destructive pancreatitis, the insufficiency of the insular apparatus was also stated.

To compose a standard approach to patient's condition assessing, taking into consideration laboratory and instrumental methods and their correlation with general clinical data, we used the following evaluation algorithm. Obser- 
Table 1. Dynamics of vital signs during treatment

\begin{tabular}{cccccc}
\hline & \multicolumn{5}{c}{ Stage of research } \\
\cline { 2 - 6 } & 1 stage & 2 stage & 3 stage & 4 stage & 5 stage \\
\hline RR $^{1}$ min. & $23,0 \pm 0,9^{*}$ & $19,4 \pm 0,7$ & $18,9 \pm 0,6$ & $17,4 \pm 0,5$ & $17,1 \pm 0,2^{*}$ \\
\hline $\mathrm{HR}^{1}, \mathrm{~min}$. & $112,7 \pm 3,2^{*}$ & $104,6 \pm 2,9$ & $94,1 \pm 4,0$ & $88,4 \pm 3,7^{*}$ & $86,4 \pm 4,5^{*}$ \\
\hline $\mathrm{SatO}_{2^{\prime}} \%$ & $95,0 \pm 0,7$ & $98,1 \pm 0,2$ & $97,7 \pm 0,4$ & $97,8 \pm 0,2$ & $97,7 \pm 0,4$ \\
\hline $\mathrm{SBP}^{1} \mathrm{~mm} \cdot \mathrm{Hg}$ & $128,0 \pm 9,9^{*}$ & $127,2 \pm 9,5$ & $121,1 \pm 5,8$ & $117,3 \pm 5,8^{*}$ & $124,6 \pm 6,3$ \\
\hline DBP $^{1} \mathrm{~mm} \cdot \mathrm{Hg}$ & $79,6 \pm 5,2$ & $77,7 \pm 5,8$ & $76,4 \pm 5,6$ & $76,1 \pm 4,2$ & $74,7 \pm 4,2$ \\
\hline Body temperature.Co & $37,8 \pm 0,5$ & $38,1 \pm 0,4$ & $37,5 \pm 0,5$ & $37,1 \pm 0,4$ & $37,1 \pm 0,2$ \\
\hline Diuresis, $\mathrm{ml}$ & $825 \pm 63^{*}$ & $1180 \pm 194$ & $1784 \pm 112$ & $2045 \pm 220$ & $1959 \pm 517^{*}$ \\
\hline
\end{tabular}

* - the difference is significant compared to the norm $(p<0,05)$

RR- respiration rate; Sat02 -blood saturation; SBP - systolic blood pressure; DBP - diastolic blood pressure; HR-heart rate

Table 2. Dynamics of biochemistry parameters

\begin{tabular}{cccccc}
\hline \multirow{2}{*}{ Indicator } & \multicolumn{5}{c}{ Stage of research } \\
\cline { 2 - 6 } & 1 stage & 2 stage & 3 stage & 4 stage & 5 stage \\
\hline Total protein, $\mathrm{g} / \mathrm{l}$ & $70,0 \pm 0,7^{*}$ & $64,1 \pm 0,2$ & $47,5 \pm 0,4$ & $40,1 \pm 0,2$ & $46,6 \pm 0,4^{*}$ \\
\hline Glucose, $\mathrm{mmol} / \mathrm{I}$ & $5,86 \pm 0,3^{*}$ & $9,13 \pm 0,5^{*}$ & $8,71 \pm 0,4^{*}$ & $7,50 \pm 0,4$ & $9,98 \pm 0,7^{*}$ \\
\hline Urea $\mathrm{mmol} / \mathrm{I}$ & $8,71 \pm 0,3^{*}$ & $10,92 \pm 0,2$ & $8,3 \pm 0,5$ & $10,8 \pm 0,4$ & $14,1 \pm 0,3^{*}$ \\
\hline Creatininemmol/l & $95,0 \pm 0,2$ & $121,1 \pm 0,7$ & $134,2 \pm 0,5$ & $123,4 \pm 0,3$ & $113,6 \pm 0,4$ \\
\hline Bilirubin $\mathrm{mmol} / \mathrm{I}$ & $19,4 \pm 0,6^{*}$ & $11,84 \pm 0,4$ & $9,0 \pm 0,8^{*}$ & $9,3 \pm 0,7$ & $18,0 \pm 0,6$ \\
\hline
\end{tabular}

${ }^{*}$-the difference is significant compared to the norm $(p<0,05)$

Table 3. Dynamics of hematology parameters

\begin{tabular}{cccccc}
\hline \multirow{2}{*}{ Indicator } & \multicolumn{5}{c}{ Stage of research } \\
\cline { 2 - 6 } & $\mathbf{1}$ stage & 2 stage & 3 stage & 4 stage & 5 stage \\
\hline Hemoglobin, $\mathrm{g} / \mathrm{l}$ & $169,3 \pm 0,4^{*}$ & $156,5 \pm 0,3$ & $129,3 \pm 0,7$ & $112,2 \pm 0,2$ & $92,5 \pm 0,8^{*}$ \\
\hline Leukocytes, $10^{9} / \mathrm{I}$ & $15,0 \pm 1,2^{*}$ & $10,2 \pm 0,9$ & $14,41 \pm 0,1$ & $16,28 \pm 0,2$ & $9,1 \pm 0,5^{*}$ \\
\hline Immature neutrophils, \% & $5,5 \pm 1,4^{*}$ & $4,5 \pm 1,1$ & $6,2 \pm 1,6$ & $11 \pm 1,5^{*}$ & $9,8 \pm 0,4^{*}$ \\
\hline Lymphocytes, \% & $21,6 \pm 3,2^{*}$ & $17,7 \pm 3,6$ & $17,9 \pm 2,4$ & $17,1 \pm 2,1$ & $15,3 \pm 1,9^{*}$ \\
\hline Fibrinogen & $3,4 \pm 0,3$ & $4,8 \pm 0,7$ & $4,9 \pm 0,6$ & $7,11 \pm 0,5$ & $4,8 \pm 0,3$ \\
\hline Prothrombin index & $78,02 \pm 0,3$ & $78,11 \pm 1,1$ & $85,48 \pm 2,4$ & $78,94 \pm 1,6$ & $79,15 \pm 0,9$ \\
\hline
\end{tabular}

*-the difference is significant compared to the norma $(p<0,05)$

vation of patients was carried out in five stages: 1 st - initial stage (hospitalization of the patient); 2 nd stage -1 day after the start of intensive care; 3 rd, 4 th and 5 th - the corresponding day of the disease (assessment of the patient's condition and the effectiveness of drug therapy). Descriptive statistics methods were used to describe the complex of primary data. The difference was considered significant and statistically significant at $\mathrm{p}<0.05$. (Tab.I)

At the time of patients hospitalization, the mean systolic arterial pressure (SAP) was $(128.0 \pm 9.9) \mathrm{mmHg}$, with severe tachycardia $(112.7 \pm 3.2) \mathrm{min}$. and tachypnea $(23.0 \pm 0.9) \mathrm{min}$. By the end of the second day, as a result of the therapy, the majority of patients showed a tendency to a decrease in the SAP to the levels of (121.1 $\pm 5.8) \mathrm{mm} \mathrm{Hg}$, which was largely due to the conducted infusion therapy.
The normalization of SatO2 is also noteworthy, which is largely due to the leveling of stress-gene factors (pain, providing the body with energy donors, correction of metabolic disorders, support for active protein mass, prevention of background immunosuppression).

According to the data presented in table 2, it is possible to draw preliminary conclusions that in patients with AP, signs of intoxication tend to increase. According to the results of biochemical studies, an increase in the level of urea was observed from $8.71 \pm 0.3$ to $14.1 \pm 0.3 \mathrm{mmol} / \mathrm{L}$ on the background of progressive hypoproteinemia (at the time of hospitalization, $70.0 \pm 0.7 \mathrm{~g} / \mathrm{L}$ ), while on the fifth day of treatment, the level of blood proteins decreased to levels of $46.6 \pm 0.4 \mathrm{~g} / \mathrm{l}$. (Tab. II)

According to peripheral blood examination results during 1-3 day of treatment, the signs of dehydration (hemoglobin $169.3 \pm 0.4 \mathrm{~g} / \mathrm{l})$ with a reliable $(\mathrm{p}<0.05)$ with a 

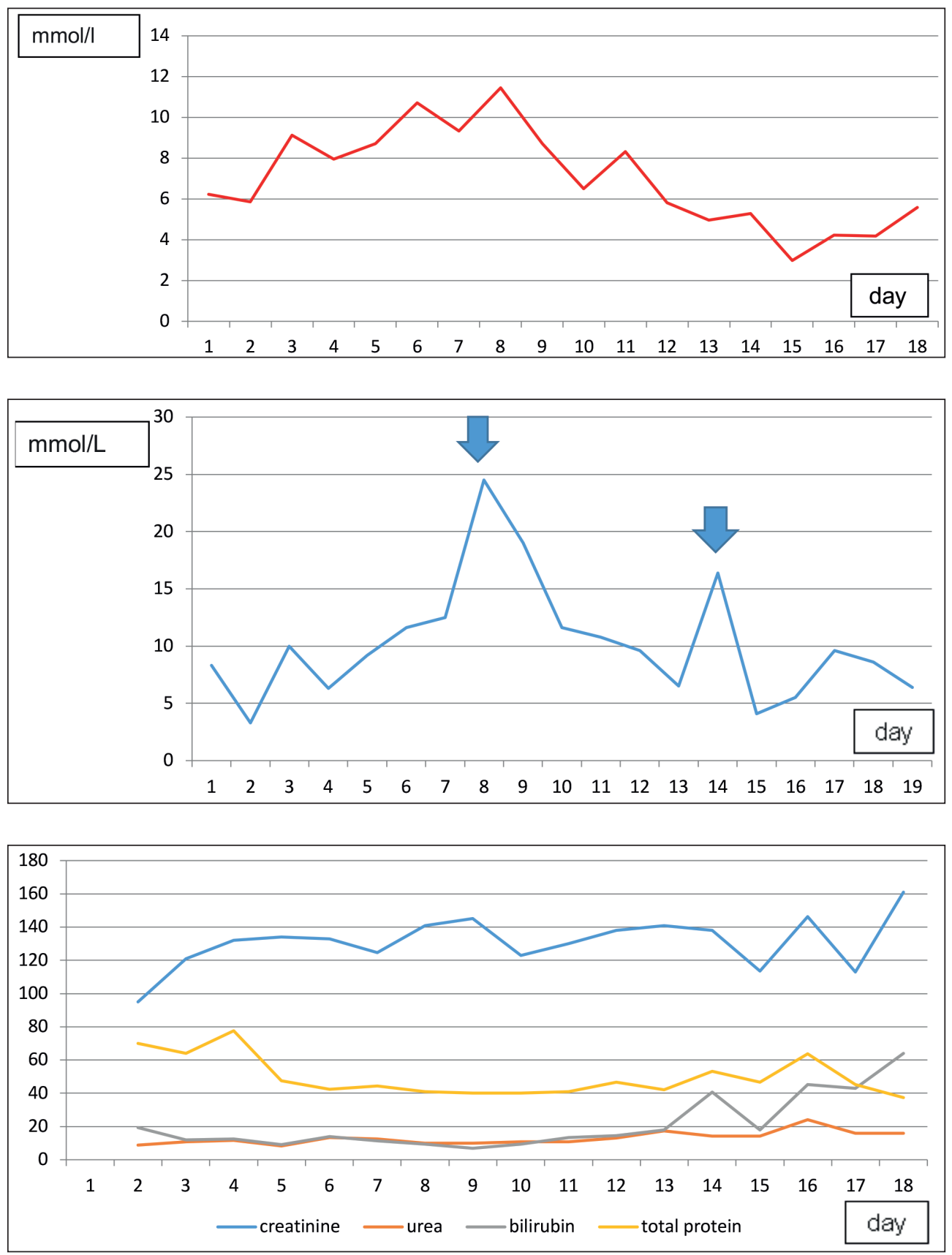

Fig. 2. Dynamics of glucose levels.

Fig.3. Dynamics of glucose levels according surgery treatment.

Fig.4. Dynamics of biochemical parameters. tendency to its level decrease during treatment were stated $(92.5 \pm 0.8 \mathrm{~g} / \mathrm{l})$. The majority of patients had confirmed signs of a systemic inflammatory reaction: a quantitative increase in the leukocytes level $(16.28 \pm 0.2 \times 109$ / L) in peripheral blood and stab neutrophils $(11.0 \pm 1.5 \%)$ on the background of a lymphocytes level decrease ( $15.3 \pm$ 1.9\%).(Tab. III)

Comparison of blood glucose levels changes at different periods of the disease with insulin content allowed us to make a conclusion that the glucose level depends on the severity of the clinical course of ACP and the function of the insulin apparatus of the pancreas, and therefore can serve as a marker of the disease clinical course severity. The dynamics of glucose levels changes in the peripheral blood serum during treatment (in days) is shown in Fig..2. The peak increase in blood glucose levels, as a rule, coincided with surgical interventions (mini-invasive and / or open surgery), which served as a kind of stress-generating factors for the patient's body in ACP (Fig.3).

In the course of analyzing the patient's condition during treatment, we monitored laboratory indicators of metabolic disorders, which, although not being triggers in the development of acute pancreatitis, however, with its further development, they become important in pathogenesis and directly determine the clinical course and consequences of the disease. During the study, we monitored the biochemical parameters (total protein and its fractions in the blood, the level of urea, creatinine, bilirubin) - (Fig.4).

Undoubtedly, the assessment of biochemical parameters in the general plan of the ACP treatment algorithm 
allowed us to determine the depth and direction of metabolic disorders, the mechanism for the development of other changes in the patient's body, and also made it possible to assess the nature of pathological changes in the pancreas. However, it is extremely difficult to clearly state that these indicators are the criteria for determining the clinical course of ACP. It should be noted that we did not find a clear correlation between these indicators in the group of examined patients, although indirectly they characterized the patient's condition and the clinical course of the disease.

In cases with severe gland edema, a moderate increase in the respiration rate $(18 \pm 2.1)$ min was detected, which is directly related to the processes of phosphorylation and the rate of ATP production. Hemorrhagic pancreatitis was accompanied by an increase in respiratory rate in active and controlled states $(20 \pm 3.4) \mathrm{min}$. on the background of intensification of ATP production, and with necrotic pancreatitis, a more pronounced increase in respiratory activity is characteristic (respiratory rate up to $24 \pm 2.8 \mathrm{~min}$.). This is usually due to the fact that hemorrhagic pancreatitis is accompanied by an increase in energy production mainly due to intensification of respiration, and with necrotic forms of the disease, a relative depression of bioenergetic processes in liver mitochondria was observed.

\section{DISCUSSION}

According to the abovementioned data, DM does not develop in all patients with AP. In acute lesions of the pancreatic gland, islet cells are often well preserved despite severe damage to acinar cells [8]. The mechanism of the relative conservation of endocrine cells compared with the destruction of exocrine parenchyma does not have a clear explanation, but may be associated with a predominant increase in the apoptosis index in acinar cells compared to islet cells. Probably due to the mechanism of blocking apoptosis $[4,8]$.

The pathogenesis of pancreatogenic diabetes is mainly explained by the development and progression of destructive changes, hypoxic changes in devitalized pancreatic tissues, and endocrine sclerosis. However, an autoimmune effect on islet cells is also possible, which correlates with morphological and functional changes in $\beta$-cells $[4,8]$.

A certain role in the pathogenesis of diabetes in ACP is played by constitutionally determined tissue resistance, which is much more common in individuals with obesity and hyperlipidemia $[14,15]$. Obesity aggravates the clinical course of the disease and complicates its prognosis.

In general, according to the results of the study, it can be noted that the clinical course of the disease, severity and prognosis of the patient's condition correlate with the dynamics of endotoxemia markers and liver failure, and among the general indicators of protein and carbohydrate metabolism, sugar level is one of the most sensitive criteria.

Thus, the determination and monitoring of the blood glucose level in patients with ACP allows early identification of the severity of the process and predicts a possible adverse course, adjusts the starting therapy, identifies the need for indications for surgical intervention and in cases of high risk of an adverse outcome, prescribes enhanced antibacterial and detoxification therapy.

\section{CONCLUSIONS}

1. The frequency of diabetes mellitus development as a result of primary inflammation of the pancreatic parenchyma in ACP has a linear dependence on the frequency and duration of the disease, and the likelihood of developing diabetes with an exacerbation of the process during $5-8$ years is $17.5 \%$.

2. The pathogenesis of pancreatogenic diabetes mellitus is associated with the destruction and sclerosis of endocrine pancreatic tissue, and in the presence of obesity, with insulin resistance.

3. Blood sugar levels in patients with ACP can be a reliable indicator of the severity of the disease course and correlates with other indicators of metabolism.

4. The incidence of diabetes with alcohol pancreatitis is twice higher than with the biliary genesis of the disease.

\section{REFERENCES}

1. Drozdinsky G., Grossman A., Shiber Sh. Factors Associated With the Development of Diabetes Mellitus Following a First Episode of Acute Pancreatitis. JOP. J Pancreas. 2018; 19(6):287-290.

2. Pendharkar S.A., Asrani V.M., Xiao A.Y., Yoon H.D. et al. Relationship between pancreatic hormones and glucose metabolism: A cross-sectional study in patients after acute pancreatitis. Am J Physiol Gastrointest Liver Physiol 2016; 311:50-58. doi: 10.1152/ ajpgi.00074.2016.

3. Solanki S.L., Barreto S.B., Saccone G.T.P. Acute pancreatitis due to diabetes: The role of hyperglycaemia and insulin resistance. 2012; 12: 234-239. doi: 10.1016/j.pan.2012.01.003 .

4. Tu J., Zhang J., Ke L., Yang Y. et al. Endocrine and exocrine pancreatic insufficiency after acute pancreatitis: long-term follow-up study. BMC Gastroenterol. 2017;17(1):114. doi:10.1186/s12876-017-0663-0 .

5. Tu J., Yang Y., Zhang J., Yang Q. et al. Effect of the disease severity on the risk of developing new-onset diabetes after acute pancreatitis. Medicine. 2018;97(22):107-113. doi: 10.1097/MD.0000000000010713.

6. Das S.L., Kennedy J.I., Murphy R., Phillips A.R. et al. between the exocrine and endocrine pancreas after acute pancreatitis. World J Gastroenterol. 2014; 20(45):17196-205. doi: 10.3748/wjg.v20. i45.17196.

7. Hari Kumar K.V.S., Sharma R., Manrai V., Sood A.K. Visceral Adipose Tissue as a Risk Factor for Diabetes Mellitus in Patients with Chronic Pancreatitis: A Cross-sectional, Observational Study. Diabetes Therapy. 2017; 8(5): 1057-1064. doi: 10.1007/s13300-017-0304-1 .

8. Malka D., Levy Ph. Acinar-islet cell interactions: Diabetes mellitus in chronic pancreatitis. Pancreatic disease: Basic science and clinical management . London. 2004:251-266.

9. Vipperla K., Papachristou G., Slivka A., Whitcomb D. et al. Risk of NewOnset Diabetes Is Determined by Severity of Acute Pancreatitis. Pancreas. 2016; 45(1):e14-e15. doi: 10.1097/MPA.0000000000000536.

10. Wu D., Xu Y., Wang X. Endocrine pancreatic function changes after acute pancreatitis. Pancreas. 2011;40(7):1006-1011. doi: 10.1097/ MPA.0b013e31821fde3f 
11. Ewald N., Bretzel R.G. Diabetes mellitus secondary to pancreatic diseases (Type 3c)-are we neglecting an important disease? Eur J Intern Med. 2013;24(3):203-6. doi: 10.1016/j.ejim.2012.12.017.

12. Srivastava B.K., Meera M., Anusha S., Mohan V. Association of acute pancreatitis in a patient with Type 1 diabetes. Journal of Diabetology. 2017;8(2):56-57. doi: 10.4103/jod.jod_16_17.

13. Huh J., Jeon H., ParkS., ChoiE.H. et al. Diabetes Mellitus is AssociatedWith Mortality in Acute Pancreatitis. Journal of Clinical Gastroenterology.2018; 52(2):178-183. doi: 10.1097/MCG.0000000000000783.

14. Ma J-H.,Yuan Y-J., Lin S-H., Pan J-Y. Nomogram for predicting diabetes mellitus after the first attack of acute pancreatitis. European Journal of Gastroenterology \& Hepatology. 2019;31(3):323-328. doi: 10.1097/ MEG.0000000000001307.

15. Farkas N., Mikó A., Garami A., Szabó I. et al. Preexisting Diabetes Elevates Risk of Local and Systemic Complications in Acute Pancreatitis: Systematic Review and Meta-analysis. Pancreas. 2018;47(8):917-923. doi: 10.1097/MPA.0000000000001122.

\section{ORCID and contributionship:}

Adrian D. Kvit: 0000-0002-5036-3606 ${ }^{A, E, F}$

Mykhaylo M. Tutka: 0000-0003-4395-8406 ${ }^{B, D}$

Volodymyr V. Kunovskiy: 0000-0003-2796-4814 C,D,E

\section{Conflict of interest:}

The Authors declare no conflict of interest.

\author{
CORRESPONDING AUTHOR \\ Adrian D. Kvit \\ Lviv Clinical Emergency Hospital \\ 57/1 Konovaltsya st., 79057 Lviv, Ukraine \\ tel:+380505324454 \\ e-mail: adrian_kvit@yahoo.pl \\ Received: 04.03 .2020 \\ Accepted: 15.10 .2020
}

A - Work concept and design, B - Data collection and analysis, C - Responsibility for statistical analysis,

D-Writing the article, $\mathbf{E}$-Critical review, $\mathbf{F}$ - Final approval of the article 